INPLASY

PROTOCOL

To cite: Qin et al. Comparison of analgesic methods after total knee arthroplasty: A network meta-analysis. Inplasy protocol 2020120006. doi: 10.37766/inplasy2020.12.0006

Received: 01 December 2020

Published: 02 December 2020

Corresponding author:

Lu Qin

qinlu19@mails.jlu.edu.cn

Author Affiliation:

Jilin University

Support: None.

Review Stage at time of this submission: Data analysis.

Conflicts of interest:

None.

\section{Comparison of analgesic methods after total knee arthroplasty: A network meta-analysis}

Qin, L1; Zhao, S2; You, D3; Li, L4; Zhao, G5.

Review question / Objective: In this study, we compared 18 kinds of intervention measures after knee arthroplasty with network meta-analysis, in order to find out an optimal anesthesia method. There are 6 outcome indicators involved, namely visual analogue scale (VAS) score, postoperative complications, morphine consumption, function score, hospital length of stay, and patient satisfaction.

Condition being studied: The included studies are all RCT literature of the past 20 years. The patients studied are all patients after knee arthroplasty, and the quality of the included studies is mostly medium or above. The included literature involves at least one type of anesthesia for peripheral nerve block.

INPLASY registration number: This protocol was registered with the International Platform of Registered Systematic Review and Meta-Analysis Protocols (INPLASY) on 02 December 2020 and was last updated on 02 December 2020 (registration number INPLASY2020120006).

\section{INTRODUCTION}

Review question / Objective: In this study, we compared 18 kinds of intervention measures after knee arthroplasty with network meta-analysis, in order to find out an optimal anesthesia method. There are 6 outcome indicators involved, namely visual analogue scale (VAS) score, postoperative complications, morphine consumption, function score, hospital length of stay, and patient satisfaction.

Condition being studied: The included studies are all RCT literature of the past 20 years. The patients studied are all patients after knee arthroplasty, and the quality of the included studies is mostly medium or 
above. The included literature involves at least one type of anesthesia for peripheral nerve block.

\section{METHODS}

Participant or population: ASAI-III TKA patients, no race or nationality restrictions.

Intervention: $A$ total of 18 interventions were involved, including Placebo, FIB, FNB, cFNB, sFNB, ACB, SNB, ONB, PSOAS, EPI, ITM, LIA, PCA, LB, FNB+SNB, ACB+LIA, $F N B+L I A$, and PCA+FNB. Each comparison must involve at least one type of nerve block.

Comparator: A total of 18 interventions were involved, including Placebo, FIB, FNB, cFNB, sFNB, ACB, SNB, ONB, PSOAS, EPI, ITM, LIA, PCA, LB, FNB+SNB, ACB+LIA, FNB+LIA, and PCA+FNB. Each comparison must involve at least one type of nerve block.

\section{Study designs to be included: RCT.}

Eligibility criteria: (1) ASAI -III TKA patients, no race or nationality restrictions; (2) Randomised controlled trial, irrespective of language; (3) Treatment with at least one of the included 12 nerve blocks (fascia iliaca compartment block (FIB), FNB, cFNB, single femoral nerve block (sFNB), adductor canal block (ACB), sciatic nerve block (SNB), obturator nerve block (ONB), continuous posterior lumbar plexus block (PSOAS), FNB+SNB, ACB+LIA, FNB+LIA, PCA+FNB), plus other interventions, such as placebo, EPI, intrathecal morphine (ITM), LIA, PCA, and liposomal bupivacaine (LB); (4) Complete original literature and outcome measures include complete information on one of the following: VAS score (within 4, 6, 12, 24, and $48 \mathrm{~h}$ after surgery), postoperative complications (nausea and/or vomiting, urinary retention, pruritus, and sedation), function score (range of motion [ROM], Timed-Up-and-Go [TUG] test $24 \mathrm{~h}$, and TUG test $48 \mathrm{~h}$ ), hospital length of stay, morphine consumption (within $24 \mathrm{~h}$ and $48 \mathrm{~h}$ after surgery), and patient satisfaction; (5) Literature data could be used or converted into binary or continuous variables to represent indicators.

Information sources: A search strategy was formulated based on the standards established by the Cochrane Collaboration. Articles were retrieved from PubMed, Embase, The Cochrane Library, and Web of Science databases. Simultaneously, a manual search was conducted, and the articles of interest were included according to the relevant meta-research. Two researchers independently extracted the relevant data in accordance with the unified form of data extraction designed in advance. If there was any disagreement, it was settled as described above. When the data in the article were incomplete, the authors attempted to contact the corresponding author via e-mail, but no reply was received in all cases.

Main outcome(s): There are 6 outcome indicators involved, namely ,visual analogue scale (VAS) score, postoperative complications, morphine consumption, function score, hospital length of stay, and patient satisfaction.Odds ratio (OR) was used for binary variables and standardised mean difference (SMD) was used for continuous variable. RevMan 5.3 was used to evaluate the quality of the included articles, and a risk of bias plot was generated. STATA 14.0 software was utilised to produce the evidence relationship plot, forest plot, rank probability plot, and funnel plot, and to conduct corresponding statistical analyses. The node-splitting method was used as the consistency test.

Quality assessment / Risk of bias analysis: RevMan 5.3 was used to evaluate the quality of the included articles, and a risk of bias plot was generated. The 'Cochrane risk bias assessment tool' was used to evaluate the quality, including seven bias items: random sequence generation (selection bias), allocation consideration (selection bias), blinding of participants and person (performance bias), blinding of outcome assessment (detection bias), incomplete outcome data (attachment bias), Each item gives its own judgment, 
and finally gets the risk of bias graph and risk of bias summary

Strategy of data synthesis: Two researchers independently extracted the relevant data in accordance with the unified form of data extraction designed in advance. If there was any disagreement, it was settled as described above. When the data in the article were incomplete, the authors attempted to contact the corresponding author via e-mail, but no reply was received in all cases. When the standard deviation was missing and since the authors could not be contacted, range or median estimation or the method described in "The Cochrane Handbook for Systematic Reviews of Interventions" was used for conversion, and the result was estimated using the confidence interval $(\mathrm{Cl})$ in accordance with the article by Hou Xiaowen and others.

Subgroup analysis: None.

Sensibility analysis: None.

Language: English.

Country(ies) involved: China.

Keywords: analgesia; network metaanalysis; pain management, peripheral nerve block; total knee arthroplasty.

Contributions of each author:

Author 1 - Lu Qin is mainly responsible for data collection, software operation and original manuscript writing.

Email: 1484734011@qq.com

Author 2 - Shishun Zhao provided statistical expertise and resources.

Email: zhaoss@jlu.edu.cn

Author 3 - Di You contributed to the development of the selection criteria and data collation.

Email: youdi@mails.jlu.edu.cn

Author 4 - Longyun Li - The author provided resources and feedback.

Email: longyun@jlu.edu.cn

Author 5 - Guoqing Zhao - The author provided feedback and approved the final manuscript.

Email: zhaoss@jlu.edu.cn 